PROCEEDINGS OF THE

AMERICAN MATHEMATICAL SOCIETY

Volume 137, Number 2, February 2009, Pages 473-478

S 0002-9939(08)09506-3

Article electronically published on August 20, 2008

\title{
REMARK ON ELLIPTIC UNITS IN A $\mathbb{Z}_{p}$-EXTENSION OF AN IMAGINARY QUADRATIC FIELD
}

\author{
TSUYOSHI ITOH
}

(Communicated by Ken Ono)

\begin{abstract}
We shall study the group of units modulo the group of elliptic units in a $\mathbb{Z}_{p}$-extension of an imaginary quadratic field.
\end{abstract}

\section{MAIN RESULt}

We fix an imaginary quadratic field $k$ which is different from $\mathbb{Q}(\sqrt{-1}), \mathbb{Q}(\sqrt{-3})$, and an odd prime number $p$. Let $\mathfrak{p}$ be a prime ideal of $k$ lying above $p$, and $K / k$ a $\mathbb{Z}_{p}$-extension which is unramified outside $\mathfrak{p}$. Assume that $\mathfrak{p}$ is totally ramified in $K / k$. For a positive integer $n$, we denote by $k_{n}$ the $n^{\text {th }}$ layer of $K / k$. Let $A_{n}$ be the Sylow $p$-subgroup of the ideal class group of $k_{n}, E_{n}$ the group of units in $k_{n}$, and $\mathfrak{p}_{n}$ the unique prime ideal of $k_{n}$ lying above $\mathfrak{p}$. Let $c\left(\mathfrak{p}_{n}\right)$ be the ideal class of $k_{n}$ which contains $\mathfrak{p}_{n}$. We put $D_{n}=A_{n} \cap\left\langle c\left(\mathfrak{p}_{n}\right)\right\rangle$ and $A_{n}^{\prime}=A_{n} / D_{n}$. Moreover, put $k_{0}=k$, and define $A_{0}, D_{0}$, and $A_{0}^{\prime}$ similarly. For a finite set $S$, we denote by $|S|$ the number of elements in $S$.

For any integer $n \geq 1$, let $\Phi_{n}$ be the group of certain elliptic units in $k_{n}$ which is defined in Section 2. We will see later that $\Phi_{n}$ has finite index in $E_{n}$. Let $B_{n}$ be the Sylow $p$-subgroup of $E_{n} / \Phi_{n}$. In this paper, we shall show the following:

Theorem 1.1. If $\left|A_{n}\right|$ is bounded as $n \rightarrow \infty$ (i.e. both of the Iwasawa $\lambda$ - and $\mu$ invariants of $K / k$ are zero), then $A_{n}^{\prime}$ and $B_{n}$ are isomorphic as $\operatorname{Gal}\left(k_{n} / k\right)$-modules for all sufficiently large $n$.

We mention that a similar result is already given in [7 for the case that $p \geq 5$ splits in $k, k_{n}$ is the ray class field of $k$ modulo $\mathfrak{p}^{n+1}$, and $\mathfrak{p}$ does not split in the absolute class field of $k$. (When $k$ is a real abelian field and $K / k$ is the cyclotomic $\mathbb{Z}_{p}$-extension, similar results are previously known. See [11], [15], etc.)

In Section 5, we will give an additional result. This result is obtained as a corollary of known results.

\section{Group of ELLIPTIC Units}

Fix an integer $n \geq 1$. In this section, we will define the group $\Phi_{n}$ of elliptic units in $k_{n}$. Our construction is similar to that of [7]. We use the same notation as given in Oukhaba [14.

Received by the editors September 4, 2007, and, in revised form, January 23, 2008.

2000 Mathematics Subject Classification. Primary 11R23; Secondary 11G16.

(C)2008 American Mathematical Society 
Let $\mathfrak{f}_{n}$ be the conductor of $k_{n} / k$, and $f_{n}$ the minimal positive integer which is contained in $\mathfrak{f}_{n} \cap \mathbb{Z}$. Note that $\mathfrak{f}_{n}$ is a positive power of $\mathfrak{p}$. Let $k_{\mathfrak{f}_{n}}$ be the ray class field of $k$ modulo $\mathfrak{f}_{n}$. We fix a $\mathbb{Z}$-basis $\left(\omega_{1}, \omega_{2}\right)$ of $\mathfrak{f}_{n}$ satisfying $\operatorname{Im}\left(\omega_{1} / \omega_{2}\right)>0$. Let

$$
\varphi_{\mathfrak{f}_{n}}:=\left(\kappa\left(1, \mathfrak{f}_{n}\right) \eta\left(\omega_{1} / \omega_{2}\right)^{2} \omega_{2}^{-1}\right)^{12 f_{n}}
$$

be the Siegel-Ramachandra-Robert invariant defined in [14, Definition 2], where $\kappa\left(t, \mathfrak{f}_{n}\right)$ is the Klein form (see [13, p. 27]) and

$$
\eta(\tau)=e^{2 \pi i \tau / 24} \prod_{n=1}^{\infty}\left(1-e^{2 \pi i n \tau}\right)
$$

is the Dedekind eta function.

As noted in [14], $\varphi_{\mathfrak{f}_{n}}$ coincides with $E\left(\mathfrak{c}_{0}\right)$ in [19. We also note that $\varphi_{\mathfrak{f}_{n}}$ is only dependent on $\mathfrak{f}_{n}$ (see [19, p. 223]). By [14, Proposition 2] or [19], we know that $\varphi_{\mathfrak{f}_{n}}$ is an algebraic integer in $k_{f_{n}}$ and any $12 f_{n}^{\text {th }}$ root is contained in a certain abelian extension field of $k$. We put $\tilde{\varphi}_{k_{n}, \mathfrak{f}_{n}}=N_{k_{f_{n}} / k_{n}} \varphi_{\mathrm{f}_{n}}^{2}$.

We mention that the roots of unity contained in $k_{n}$ are only \pm 1 . Hence by [19, Lemma 6], there is a unique element $u_{n}$ of $k_{n}$ which satisfies

$$
u_{n}^{3 f_{n}}=\tilde{\varphi}_{k_{n}, f_{n}} .
$$

(Note that $f_{n}$ is odd.) We also note that $u_{n}$ is a $\mathfrak{p}_{n}$-unit in $k_{n}$ (which follows from, e.g., [14, Corollary 2]). Let $E_{n}^{\prime}$ be the group of $\mathfrak{p}_{n}$-units in $k_{n}$.

Definition 2.1. Let $\Phi_{n}^{\prime}$ be the $\mathbb{Z}\left[\operatorname{Gal}\left(k_{n} / k\right)\right]$-submodule of $E_{n}^{\prime}$ generated by \pm 1 and $u_{n}$. Similarly, let $\Omega_{n}^{\prime}$ be the $\mathbb{Z}\left[\operatorname{Gal}\left(k_{n} / k\right)\right]$-submodule of $E_{n}^{\prime}$ generated by \pm 1 and $\tilde{\varphi}_{k_{n}, \mathfrak{f}_{n}}$. Moreover, we put $\Phi_{n}=E_{n} \cap \Phi_{n}^{\prime}$ and $\Omega_{n}=E_{n} \cap \Omega_{n}^{\prime}$.

By the analytic class number formula ([13, Chapter 13, Theorem 2.1], 14, Theorem A and Proposition 16]), we obtain

$$
\left(E_{n}: \Omega_{n}\right)=\frac{h\left(k_{n}\right)}{h(k)}\left(24 f_{n}\right)^{p^{n}-1}
$$

where $h\left(k_{n}\right)$ is the class number of $k_{n}$ and $h(k)$ is the class number of $k$. By the definition of $\Phi_{n}$, we obtain the following:

\section{Lemma 2.2.}

$$
\left(E_{n}: \Phi_{n}\right)=\frac{h\left(k_{n}\right)}{h(k)} 8^{p^{n}-1} .
$$

Let $f$ be a homomorphism $E_{n} / \Phi_{n} \rightarrow E_{n}^{\prime} / \Phi_{n}^{\prime}$ induced from the natural mapping. Since $E_{n} \cap \Phi_{n}^{\prime}=\Phi_{n}$, we see that $f$ is injective.

Lemma 2.3. Assume that $\left|A_{n}\right|$ is bounded as $n \rightarrow \infty$. If $n$ is sufficiently large, then the cokernel of $f$ is finite and its order is prime to $p$. 
Proof. Let Coker $(f)$ be the cokernel of $f$. We have the following commutative diagram of exact sequences:

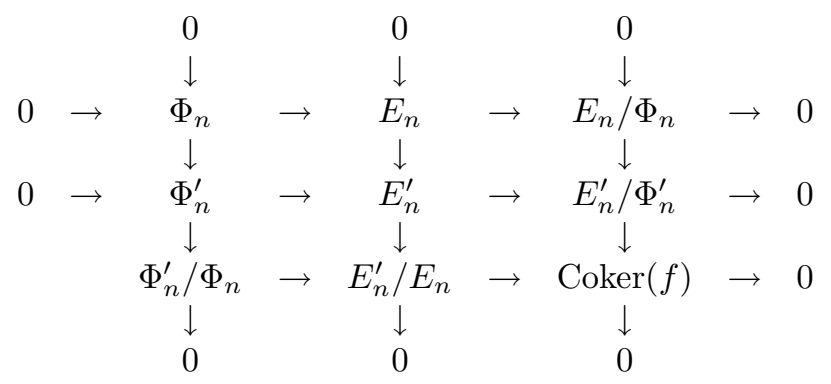

Let $d$ be the order of the ideal class of $k_{n}$ which contains $\mathfrak{p}_{n}$. We fix an algebraic integer $v$ of $k_{n}$ which satisfies $\mathfrak{p}_{n}^{d}=(v)$. By using [14, Corollary 2], we can see that $\mathfrak{p}_{n}^{24 h(k)}=\left(u_{n}\right)$ and hence $24 h(k)$ is divisible by $d$.

Since $\left|A_{n}\right|$ is bounded as $n \rightarrow \infty$, we can see that $A_{n}^{\mathrm{Gal}\left(k_{n} / k\right)}=D_{n}$ for all sufficiently large $n$ (cf. [5, Theorem 2], 3, Proposition 2.2]). By the genus formula, we have $\left|A_{n}^{\operatorname{Gal}\left(k_{n} / k\right)}\right|=\left|A_{0}\right|$. If $n$ is sufficiently large, we get $\left|A_{0}\right|=\left|D_{n}\right|$ and then $24 h(k) / d$ is prime to $p$. We note that $u_{n} \Phi_{n}$ is a generator of $\Phi_{n}^{\prime} / \Phi_{n}$ and $v E_{n}$ is a generator of $E_{n}^{\prime} / E_{n}$. Since $v^{24 h(k) / d} E_{n}=u_{n} E_{n}$, we see that the order of the cokernel of the mapping $\Phi_{n}^{\prime} / \Phi_{n} \rightarrow E_{n}^{\prime} / E_{n}$ is finite and prime to $p$.

\section{Proof of Theorem 1.1}

Assume that $\left|A_{n}\right|$ is bounded as $n \rightarrow \infty$. Let $B_{n}$ be the Sylow $p$-subgroup of $E_{n} / \Phi_{n}$, and $B_{n}^{\prime}$ the Sylow $p$-subgroup of $E_{n}^{\prime} / \Phi_{n}^{\prime}$. By Lemma 2.3 , we have $B_{n} \cong B_{n}^{\prime}$ if $n$ is sufficiently large.

The proof of Theorem 1.1 is given by using a well-known argument (cf. [11, [15], 7], etc.). Fix a positive integer $n$ which satisfies $B_{l} \cong B_{l}^{\prime}$ and $\left|A_{l}\right|=\left|A_{n}\right|$ for all $l \geq n$. We can take a positive integer $m>n$ which satisfies

$$
\operatorname{ker}\left(A_{n}^{\prime} \rightarrow A_{m}^{\prime}\right)=A_{n}^{\prime}
$$

We put $\Gamma_{m, n}=\operatorname{Gal}\left(k_{m} / k_{n}\right)$

From the results given in Section 2, we see that $\Phi_{m}^{\prime} /\{ \pm 1\}$ is a free rank one $\mathbb{Z}\left[\operatorname{Gal}\left(k_{m} / k_{0}\right)\right]$-module. Hence both of the Tate cohomology groups $\hat{H}^{0}\left(\Gamma_{m, n}, \Phi_{m}^{\prime}\right)$ and $H^{1}\left(\Gamma_{m, n}, \Phi_{m}^{\prime}\right)$ are trivial. By using [14, Proposition 3], we can see that $N_{k_{m} / k_{n}} \Phi_{m}^{\prime}=\Phi_{n}^{\prime}$. From this, we see that $\left(\Phi_{m}^{\prime}\right)^{\Gamma_{m, n}}=\Phi_{n}^{\prime}$ because $\hat{H}^{0}\left(\Gamma_{m, n}, \Phi_{m}^{\prime}\right)$ is trivial.

By taking the long cohomology sequence of the following exact sequence

$$
0 \rightarrow \Phi_{m}^{\prime} \rightarrow E_{m}^{\prime} \rightarrow E_{m}^{\prime} / \Phi_{m}^{\prime} \rightarrow 0,
$$

we obtain the following exact sequence:

$$
0 \rightarrow \Phi_{n}^{\prime} \rightarrow E_{n}^{\prime} \rightarrow\left(E_{m}^{\prime} / \Phi_{m}^{\prime}\right)^{\Gamma_{m, n}} \rightarrow H^{1}\left(\Gamma_{m, n}, \Phi_{m}^{\prime}\right) .
$$

Since $H^{1}\left(\Gamma_{m, n}, \Phi_{m}^{\prime}\right)$ is trivial, we see that $B_{n}^{\prime} \cong\left(B_{m}^{\prime}\right)^{\Gamma_{m, n}}$. By using Lemma 2.2, we obtain

$$
\left|B_{n}^{\prime}\right|=\left|B_{n}\right|=\frac{\left|A_{n}\right|}{\left|A_{0}\right|}=\frac{\left|A_{m}\right|}{\left|A_{0}\right|}=\left|B_{m}\right|=\left|B_{m}^{\prime}\right|,
$$


and then we have an isomorphism $B_{n}^{\prime} \cong B_{m}^{\prime}$ induced from the natural injection. Hence the action of $\Gamma_{m, n}$ on $B_{m}^{\prime}$ is trivial. Consequently,

$$
H^{1}\left(\Gamma_{m, n}, B_{m}^{\prime}\right) \cong B_{m}^{\prime} \cong B_{n}^{\prime} \cong B_{n} .
$$

On the other hand, we obtain the isomorphism

$$
H^{1}\left(\Gamma_{m, n}, E_{m}^{\prime}\right) \cong H^{1}\left(\Gamma_{m, n}, B_{m}^{\prime}\right)
$$

by taking the exact sequence of the Tate cohomology groups of (3.1). Moreover, we can see that

$$
H^{1}\left(\Gamma_{m, n}, E_{m}^{\prime}\right) \cong \operatorname{ker}\left(A_{n}^{\prime} \rightarrow A_{m}^{\prime}\right)
$$

by using the same argument given in the proof of [9, Theorem 12]. Since $\operatorname{ker}\left(A_{n}^{\prime} \rightarrow\right.$ $\left.A_{m}^{\prime}\right)=A_{n}^{\prime}$, we have shown Theorem 1.1.

\section{Consideration For Theorem 1.1}

Assume that $p$ splits in $k$. Let $\mathfrak{p}$ be a prime of $k$ lying above $p$ and $K / k$ the unique $\mathbb{Z}_{p}$-extension unramified outside $\mathfrak{p}$. Moreover, we assume that $\mathfrak{p}$ is totally ramified in $K / k$. Fukuda and Komatsu studied this $\mathbb{Z}_{p}$-extension in 3 . By using [3. Proposition 2.2], we see that if $\left|A_{0}\right|=\left|D_{0}\right|$, then $\left|A_{n}\right|$ is bounded as $n \rightarrow \infty$. In this case, we can see that $\left|A_{n}^{\prime}\right|=1$ for all $n$, and hence Theorem 1.1 is trivially satisfied. However, Fukuda and Komatsu also found many imaginary quadratic fields such that $\left|A_{0}\right| \neq\left|D_{0}\right|$ and satisfy the assumption of Theorem 1.1 (see [3]). This implies there are nontrivial examples for Theorem 1.1 in this case.

Assume that $p$ does not split in $k$. In this case, if $p$ does not divide the class number of $k$ (i.e. $A_{0}$ is trivial), then for any $\mathbb{Z}_{p}$-extension $K / k$ and for any $n$, $A_{n}$ is trivial. We also remark that if the class number of $k$ is divisible by $p$, then the cyclotomic $\mathbb{Z}_{p}$-extension of $k$ does not satisfy the assumption of Theorem 1.1 because $\left|A_{n}\right|$ is not bounded. However, Ozaki's result [17, Theorem 2] tells us that if "Greenberg's generalized conjecture" 6, Conjecture 3.5] holds for $k$ and $p$, then there are infinitely many $\mathbb{Z}_{p}$-extensions of $k$ which satisfy the assumption of Theorem 1.1.

\section{Additional Result}

Let the notation be as in the previous sections. In this section, we assume that $p$ does not split in $k$, and $K / k$ is the cyclotomic $\mathbb{Z}_{p}$-extension. We noted in Section 4 that we cannot apply Theorem 1.1 for $K / k$ except for the trivial case. However, we can obtain a similar type result. Kubert and Lang 12 pointed out that the group of "circular numbers" modulo the group of "modular numbers" relates to the Stickelberger ideal. We shall use their idea.

For a positive integer $r$, we put $\zeta_{r}=e^{2 \pi i / r}$. Fix an integer $n \geq 1$. Let $\mathbb{Q}_{n}$ be the $n^{\text {th }}$ layer of the cyclotomic $\mathbb{Z}_{p}$-extension of $\mathbb{Q}$. Note that the maximal real subfield of $k_{n}$ is $\mathbb{Q}_{n}$. We put $\Gamma_{n}=\operatorname{Gal}\left(\mathbb{Q}_{n} / \mathbb{Q}\right)$. Let $C_{n}^{\prime}$ be the $\mathbb{Z}\left[\Gamma_{n}\right]$-module generated by \pm 1 and

$$
N_{\mathbb{Q}\left(\zeta_{p^{n+1}}\right) / \mathbb{Q}_{n}}\left(1-\zeta_{p^{n+1}}\right) .
$$

Let $\chi$ be the Dirichlet character corresponding to $k$ and $d$ the conductor of $k$. We denote by $q_{n}$ the least common multiple of $d$ and $p^{n+1}$. We put

$$
\xi_{n}(\chi)=-\frac{1}{q_{n}} \sum_{0<a<q_{n},\left(a, q_{n}\right)=1} a \chi(a)\left(\left.\sigma_{a}\right|_{\mathbb{Q}_{n}}\right)^{-1},
$$


where $\sigma_{a}$ is the element of $\operatorname{Gal}\left(\mathbb{Q}\left(\zeta_{q_{n}}\right) / \mathbb{Q}\right)$ defined by $\zeta_{q_{n}}^{\sigma_{a}}=\zeta_{q_{n}}^{a}$ (see, e.g., [20]). It is well known that

$$
2 \sum_{0<a<q_{n},\left(a, q_{n}\right)=1}\left(\frac{1}{2}-\frac{a}{q_{n}}\right)\left(\left.\sigma_{a}\right|_{k_{n}}\right)^{-1}
$$

is contained in $\mathbb{Z}\left[\operatorname{Gal}\left(k_{n} / \mathbb{Q}\right)\right]$ (see, e.g., [2, Theorem 1 (i)], [18, Theorem 7.2.2]). From this, we can see that $\xi_{n}(\chi)$ is contained in $\mathbb{Z}\left[\Gamma_{n}\right]$.

Let $u_{n}$ be the element of $k_{n}$ defined in Section 2. By using the result of Gillard [4] (which is a generalization of the result of Kubert and Lang [12]), we can show that

$$
u_{n}=\left(N_{\mathbb{Q}\left(\zeta_{p^{n+1}}\right) / \mathbb{Q}_{n}}\left(1-\zeta_{p^{n+1}}\right)\right)^{2 \xi_{n}(\chi)}
$$

(See [4, p. 184, Corollaire]. See also [10].) Hence we see that $\Phi_{n}^{\prime}$ is contained in $C_{n}^{\prime}$, and

$$
\left(C_{n}^{\prime} / \Phi_{n}^{\prime}\right) \otimes_{\mathbb{Z}} \mathbb{Z}_{p} \cong \mathbb{Z}_{p}\left[\Gamma_{n}\right] / \xi_{n}(\chi) \mathbb{Z}_{p}\left[\Gamma_{n}\right] .
$$

On the other hand, if $A_{0}$ is a cyclic group, then

$$
A_{n} \cong \mathbb{Z}_{p}\left[\Gamma_{n}\right] / \xi_{n}(\chi) \mathbb{Z}_{p}\left[\Gamma_{n}\right]
$$

(see [1, Lemma 2.14 and Lemma 2.15]). Hence we have obtained the following:

Theorem 5.1. If $A_{0}$ is a cyclic group, then

$$
A_{n} \cong\left(C_{n}^{\prime} / \Phi_{n}^{\prime}\right) \otimes_{\mathbb{Z}} \mathbb{Z}_{p}
$$

for all $n \geq 1$.

We note that similar type results are given in [8], [16], and [7].

\section{ACKNOWLEDGEMENTS}

The author would like to express his thanks to Miho Aoki and Taku Hongou for their comments. The author also would like to express his gratitude to Professor Keiichi Komatsu for giving useful suggestions during the collaborative work on the previous paper 7 .

\section{REFERENCES}

[1] J. Coates and S. Lichtenbaum : On l-adic zeta functions, Ann. of Math. (2) 98 (1973), 498-550. MR0330107(48:8445)

[2] J. Coates and W. Sinnott : Integrality properties of the values of partial zeta functions, Proc. London Math. Soc. (3) 34 (1977), 365-384. MR0439815 (55:12697)

[3] T. Fukuda and K. Komatsu : Noncyclotomic $\mathbb{Z}_{p}$-extensions of imaginary quadratic fields, Experiment. Math. 11 (2002), 469-475. MR 1969639 (2004g:11097)

[4] R. Gillard : Unités elliptiques et unités cyclotomiques, Math. Ann. 243 (1979), 181-189. MR:543728 (81k:12007)

[5] R. Greenberg : On the Iwasawa invariants of totally real number fields, Amer. J. Math. 98 (1976), 263-284. MR0401702 (53:5529)

[6] R. Greenberg : Iwasawa theory-past and present, Class field theory-its centenary and prospect, Adv. Stud. Pure Math., 30, 335-385, Math. Soc. Japan, Tokyo, 2001. MR 1846466 (2002f:11152)

[7] T. Itoh and K. Komatsu : On the group of modular units and the ideal class group, J. Number Theory 123 (2007), 193-203. MR.2295439

[8] K. Iwasawa : On some modules in the theory of cyclotomic fields, J. Math. Soc. Japan 16 (1964), 42-82. MR0215811 (35:6646)

[9] K. Iwasawa : On $\mathbb{Z}_{l}$-extensions of algebraic number fields, Ann. of Math. (2) 98 (1973), 246-326. MR0349627(50:2120) 
[10] D. Kersey : Modular units inside cyclotomic units, Ann. of Math. (2) 112 (1980), 361-380. MR.592295 (82h:12006)

[11] J-M. Kim, S. Bae, and I-S. Lee : Cyclotomic units in $\mathbb{Z}_{p}$-extensions, Israel J. Math. $\mathbf{7 5}$ (1991), 161-165. MR.1164588 (93i:11120)

[12] D. S. Kubert and S. Lang : Modular units inside cyclotomic units, Bull. Soc. Math. France 107 (1979), 161-178. MR545170 (81k:12006)

[13] D. S. Kubert and S. Lang : Modular units, Springer-Verlag, New York, Heidelberg, Berlin, 1981. MR648603 (84h:12009)

[14] H. Oukhaba : Index formulas for ramified elliptic units, Compositio Math. 137 (2003), 1-22. MR1981934 (2004b:11085)

[15] M. Ozaki : On the cyclotomic unit group and the ideal class group of a real abelian number field, J. Number Theory 64 (1997), 211-222. MR.1453211 (98c:11121)

[16] M. Ozaki : On the cyclotomic unit group and the ideal class group of a real abelian number field. II, J. Number Theory 64 (1997), 223-232. MR.1453211(98c:11121)

[17] M. Ozaki : Iwasawa invariants of $\mathbb{Z}_{p}$-extensions over an imaginary quadratic field, Class field theory - its centenary and prospect, Adv. Stud. Pure Math., 30, 387-399, Math. Soc. Japan, Tokyo, 2001. MR1846467 (2002e:11147)

[18] V. P. Snaith : Algebraic K-groups as Galois modules, Birkhäuser Verlag, Basel, Boston, Berlin, 2002. MR.1897817 (2003c:11149)

[19] H. M. Stark : L-functions at $s=1 . I V$, Adv. in Math. 35 (1980), 197-235. MR.563924 (81f:10054)

[20] L. C. Washington : Introduction to cyclotomic fields, 2nd edition, Springer-Verlag, New York, Berlin, Heidelberg, 1997. MR1421575 (97h:11130)

College of Science and Engineering, Ritsumeikan University, 1-1-1 Noji Higashi, Kusatsu, Shiga, 525-8577, JaPAN

E-mail address: tsitoh@se.ritsumei.ac.jp 